\title{
Diversity Enhancement of MIMO System with GA Optimized Linear Precoding of Orthogonal Space-Time Block Codes
}

\author{
Harsha Gurdasani ${ }^{1}$, A G Ananth ${ }^{2}$, N Thangadurai ${ }^{3}$ \\ ${ }^{1}$ Research Scholar, Department of E \& C E, JAIN (Deeemed -to -be University), Bangalore, India \\ ${ }^{2}$ Professor, E\&C Department, NMAMIT, Nitte, Mangalore, India \\ ${ }^{3}$ Professor, Department of E\& C E, JAIN (Deemed-to-be University), Bangalore, India.
}

\begin{tabular}{l} 
Article Info \\
\hline Article history: \\
Received Jul 14, 2020 \\
Revised May 30, 2021 \\
Accepted Jun 14, 2021 \\
\hline Keywords: \\
Alamouti, \\
BEP STBC, \\
OSTBC, \\
GA, \\
MMSE, \\
ZF \\
\hline
\end{tabular}

\begin{abstract}
The MIMO (Multiple-Input Multiple-Output) systems in the field of digital communications make it possible to improve data transmission according to two main parameters which are often antagonistic: the information rate and the transmission reliability estimated in average binary error probability (BEP) term. With such systems, CSI (Channel State Information) on transmission is a key point for reducing the BEP thanks to different power allocation strategies. Thus, a linear precoder on transmission associated with a linear decoder on reception can optimize a particular criterion thanks to this information. This paper proposes a MIMO system with precoded orthogonal space-time block codes (OSTBC) optimized by Genetic Algorithm with ZF and MMSE equalization. The performance evaluation is achieved by BER vs. SNR graphs.
\end{abstract}

Copyright () 2021 Institute of Advanced Engineering and Science. All rights reserved.

\section{Corresponding Author:}

Harsha Gurdasani

Research Scholar,Department of Electronics \& Communication Engineering, JAIN (Deemed-to-be University), Bangalore, India

Email: harshagurdasani@gmail.com

\section{INTRODUCTION}

The growing demand for wireless communication services demands from future networks greater transmission capacity and better spectral and energy efficiencies. One of the widely recognized technologies for the implementation of future networks is known as Multiple Input-Multiple Output (MIMO). This technique consists in the use of multiple antennas in both the transmitter and the receiver. The space-time block codes (STBCs) associated with wireless antenna systems is the essence of this memoir. First of all, it is the recent idea of using several antennas on the program that makes us consider diversity space in a new light. This renewed interest is driven by promising performance in terms of capacity. Alamouti STBC coding is then seen as the unifying element of spatial diversity, and its potential revealed, with the standard techniques of error control coding, involving temporal diversity. The enthusiastic symbiosis of these two processes, previously dissociated, establishes the thread of our study. Diversity is a general technique to combat the attenuation and interference inherent in wireless communication channels.

We have presented an optimal method for MIMO using Genetic Algorithm having an objective of minimum bit error rate. This algorithm is a linear pre-coding alternative that transmits multiple frames of data to each user while removing inter-user interference at the same time; it is a generalization of channel investment. As the Signal-to-Noise Ratio (SNR) increases, the sum of capacities increases and the BER of the system decreases. The elimination of inter-user interference depends a lot on the knowledge of the channel, which, varies with time, and is selective in frequency, that is, in real environments, eliminating the interference, in the best case, would do so partial. It is necessary to have a return channel to send the channel status to the transmitter, so that it generates the correct pre-coding matrices and can mitigate co-channel interference. The quantitative results show the comparison of BER performance using ZF and MMSE equalization techniques. It is found that the Genetic Algorithm optimization outperforms other schemes on the basis of BER performance. 


\section{PROPOSED METHODOLOGY}

\subsection{Alamouti codes or Space-Time Block Code (STBC)}

This first family only requires knowledge of the channel on reception (Rx-CSI) and the transmitter does not know the channel. We will see different solutions using the principle of space-time block codes or Space-Time Block Code (STBC) [10], the best known of which is the Alamouti code for two transmitting antennas. The code proposed by Alamouti [11] is based on a succession of transmissions over two symbol periods and on two antennas: it then appears in both spatial and temporal dimensions. The signals transmitted are based on complex symbols resulting from modulation (conjugate or opposite). The code is defined by:

$$
C_{2}=\left(\begin{array}{cc}
s_{1} & -s_{2}^{*} \\
s_{2} & s_{1}^{*}
\end{array}\right)
$$

The symbols to be conveyed are s 1 and s 2 . This code has the particularity of being orthogonal:

$$
C_{2} C_{2}^{*}=C_{2}^{*} C_{2}=\left(\left|s_{1}\right|^{2}+\left|s_{2}\right|^{2}\right) I_{2}
$$

And on average:

$$
E\left[C_{2} C_{2}^{*}\right]=E\left[C_{2}^{*} C_{2}\right]=2 I_{2} \text { with } E\left[\left|s_{i}\right|^{2}\right]=1
$$

It is important to multiply the code by the constant to respect the limitation of average total power emitted $\mathrm{P} 0$ on all antennas within a symbol period $\sqrt{\frac{P_{0}}{2}}$. The input-output relationship in matrix form is:

$$
Y=\sqrt{\frac{P_{0}}{2}} H C_{2}+N
$$

Where $Y,\left[n_{R} \times 2\right]$, is the matrix of the samples received and $N,\left[n_{R} \times 2\right]$ is the matrix of noise. The receiver recombines the samples received after estimating the constituents of the channel matrix. It then generates two distinct signals, as defined by: $\left\{\begin{array}{l}\tilde{y}_{1}=\frac{\sum_{j=1}^{n_{R}} h_{1, j}^{*} y_{1}^{j}+h_{2, j}\left(y_{2}^{j}\right)^{*}}{\|H\|_{F}} \\ \tilde{y}_{2}=\frac{\sum_{j=1}^{n_{R}}-h_{1, j}\left(y_{2}^{j}\right)^{*}+h_{2, j}^{*} y_{1}^{j}}{\|H\|_{F}}\end{array}\right.$

Where $h_{i, j}$ represents the complex gain between the transmitting antenna $i$ and the receiving antenna $j, y_{t}^{j}$ is the useful signal of the receiving antenna $j$ at the symbol period $t$. In addition, $\|H\|_{F}$ represents the Frobenius norm of the matrix $H$ defined by:

The useful signal is defined by:

$$
\|H\|_{F}^{2}=\operatorname{trace}\left(H H^{*}\right)=\operatorname{trace}\left(H^{*} H\right)=\sum_{i}^{n_{T}} \sum_{j}^{n_{R}}\left|h_{i, j}\right|^{2}
$$

$$
\left\{\begin{array}{l}
y_{1}^{j}=\sqrt{\frac{P_{0}}{2}}\left(h_{1, j} s_{1}+h_{2, j} s_{2}\right)+n_{1}^{j} \\
y_{2}^{j}=\sqrt{\frac{P_{0}}{2}}\left(-h_{1, j} s_{2}^{*}+h_{2, j} s_{1}^{*}\right)+n_{2}^{j}
\end{array}\right.
$$

Where $n_{t}^{j}$ is the additive noise of the receiving antenna $j$ at the symbol period $t$. The expressions of equation (5) can be expanded and give the following result:

The two recombined signals take a very simple form:

$$
\left\{\begin{array}{l}
\tilde{y}_{1}=\sqrt{\frac{P_{0}}{2}} \frac{\sum_{j=1}^{n_{R}}\left\|h_{1, j}\right\|^{2}+\left\|h_{2, j}\right\|^{2}}{\|H\|_{F}} s_{1}+\frac{\sum_{j=1}^{n_{R}} h_{1, j}^{*} n_{1}^{j}+h_{2, j}\left(n_{2}^{j}\right)^{*}}{\|H\|_{F}} \\
\tilde{y}_{2}=\sqrt{\frac{P_{0}}{2}} \frac{\sum_{j=1}^{n_{R}}\left\|h_{1, j}\right\|^{2}+\left\|h_{2, j}\right\|^{2}}{\|H\|_{F}} S_{2}+\frac{\sum_{j=1}^{n_{R}} h_{2, j}^{*} n_{1}^{j}-h_{1, j}\left(n_{2}^{j}\right)^{*}}{\|H\|_{F}}
\end{array}\right.
$$

$$
\left\{\begin{array}{l}
\tilde{y}_{1}=\sqrt{\frac{P_{0}}{2}}\|H\|_{F} s_{1}+\tilde{n}_{1} \\
\tilde{y}_{2}=\sqrt{\frac{P_{0}}{2}}\|H\|_{F} s_{2}+\tilde{n}_{2}
\end{array}\right.
$$

where $\tilde{n}_{1}$ and $\tilde{n}_{2}$ are combinations of the additive noise and are AWGN if $\mathrm{N}$ is a AWGN: for a noise iid $\mathcal{N}_{\mathbb{C}}\left(0, \sigma^{2}\right)$ then $\tilde{n}_{1}$ and $\tilde{n}_{2}$ are independent and of law $\mathcal{N}_{\mathbb{C}}\left(0, \sigma^{2}\right)$. Each signal $\tilde{y}_{i}$ only depends on a symbol $s_{i}$ and can thus be used for its estimation. As a result, the Alamouti code decouples the symbols and allows for the reduction of decision tests. The system can be thought of as two SISO systems running in parallel.In addition, without knowing the channel a priori, the code ensures the same gain $\sqrt{\frac{P_{0}}{2}}\|H\|_{F}$. This feature allows the code to be channel-agnostic while still ensuring a maximum diversity order equal to the product of the antennas during transmission and reception $n_{T} \times n_{R}$ [12], here $2 \times n_{R}$ for a Rayleigh canal. On the other hand, the system can use another matrix entry: 
With the new channel matrix,

$$
\tilde{y}=G \widetilde{H}_{2} s+G \bar{n}
$$

$$
\text { If } H=\left(\begin{array}{ll}
h_{1} & h_{2}
\end{array}\right) \quad \text { then } \quad \widetilde{H}_{2}=\left(\begin{array}{cc}
h_{1} & h_{2} \\
-\operatorname{conj}\left(h_{2}\right) & \operatorname{conj}\left(h_{1}\right)
\end{array}\right)
$$

Where conj is the conjugation operation of the elements of the matrix $\left(\operatorname{conj}(x)=\left(x^{*}\right)^{T}=\left(x^{T}\right)^{*}\right)$, and the noise defined by:

$$
\text { If } \quad N=\left(\begin{array}{ll}
n_{1} & n_{2}
\end{array}\right) \quad \text { then } \quad \bar{n}=\left(\begin{array}{cc}
h_{1} & h_{2} \\
-\operatorname{conj}\left(h_{2}\right) & \operatorname{conj}\left(h_{1}\right)
\end{array}\right)
$$

The new channel matrix thus defined then has the following property:

Then just choose $\mathrm{G}$ as the suitable filter:

$$
\widetilde{H}_{2}^{*} \widetilde{H}_{2}=\|H\|_{F}^{2} I_{2}
$$

$$
G=\frac{\widetilde{H}_{2}^{*}}{\|H\|_{F}}
$$

The noise $\tilde{n}=G \bar{n}$ is a Gaussian noise of variance $\sigma_{n}^{2}$ if the noise $N$ is also an additive Gaussian noise of the same variance.

By considering either a linear recombination of the samples received, or a new channel matrix associated with an adapted filtering, the Alamouti code allows to use orthogonality to diagonalize the channel. The two symbols are decoupled and see the same channel, that is to say, the same gain and a noise of the same statistics.

However, using the code has two major drawbacks. The first is loss of flow. Indeed, an Alamouti code has the same bit rate as a SISO system but half as much as a spatial multiplexing [8]. We will note the performance of the $\mathcal{R}$ code as the number of symbols emitted over the number of symbol periods necessary for transmission. We have $\mathcal{R}=1$ for the Alamouti code and $\mathcal{R}=n_{T}$ for spatial multiplexing.

In addition, if you want to control the transmission power, the coefficient $\sqrt{\frac{P_{0}}{2}}$ is necessary. Consequently, a penalizing coefficient of $1 / 2$ or $-3 \mathrm{~dB}$ appears on the power received from the equivalent scheme compared to a SISO system [11].

\subsection{Orthogonal Space-Time Block Code (OSTBC)}

The Alamouti code is only suitable for systems with two transmitting antennas and Tarokh et al. [12, 13] generalized the orthogonal STBC (OSTBC) and this whatever $n_{T}$. The principle is to consider a train of $N_{s}$ symbols to be transmitted that the code will transmit by successive bursts over $N_{p}$ symbol periods [1]. The flow efficiency is noted:

$$
\mathcal{R}=\frac{N_{S}}{N_{p}}
$$

The bursts emitted will be the symbols or their conjugates weighted by 1 or -1 and the vectors emitted must be orthogonal. The generated code is a matrix whose two dimensions are space and time, $\left[n_{T} \times N_{p}\right]$. After calculating the channel, the connected receiver recombines the samples received and obtains signals based on only one broadcast symbol. As a result, the symbol estimates are separated.

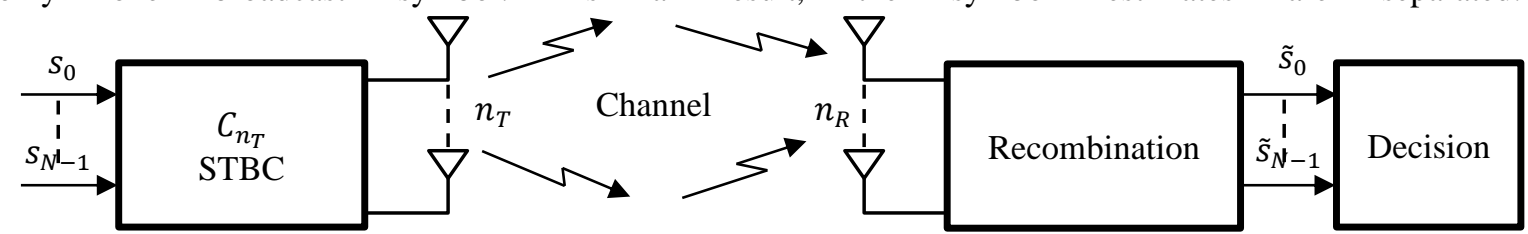

Figure 1. Diagram of the MIMO transmission using OSTBC

The orthogonality of the code allows an independent estimation of each $s_{i}$ symbol with the signal $\tilde{s}_{i}$. Figure 1 shows the OSTBC synopsis. The proposed principle is simple and very easy to set up: the compromise between performance (BER and throughput) and complexity (CSI at reception only) is interesting. In the best of cases, it is a matter of obtaining a code with the greatest possible return. For example, $\mathcal{R}=1$ for the Alamouti code. The solutions are limited and are as follows:

- $\quad$ The solution proposed by Alamouti (Equation (1)) which is the only one to obtain $\mathcal{R}=1$;

- $\quad$ OSTBC for complex modulations and for any value of $n_{T}$ but with $\mathcal{R}=\frac{1}{2}$ [13];

- $\quad$ Solutions for $n_{T}=\{3,4\}$ but with $\mathcal{R}=\frac{3}{4}$, 


$$
\begin{aligned}
& C_{3}=\left(\begin{array}{cccc}
s_{1} & -s_{2}^{*} & \frac{s_{3}^{*}}{\sqrt{2}} & \frac{s_{3}^{*}}{\sqrt{2}} \\
s_{2} & s_{1}^{*} & \frac{s_{3}^{*}}{\sqrt{2}} & -\frac{s_{3}^{*}}{\sqrt{2}} \\
\frac{s_{3}}{\sqrt{2}} & \frac{s_{3}}{\sqrt{2}} & \frac{-s_{1}-s_{1}^{*}+s_{2}-s_{2}^{*}}{2} & \frac{s_{1}-s_{1}^{*}+s_{2}+s_{2}^{*}}{2}
\end{array}\right) \\
& C_{4}=\left(\begin{array}{cccc}
s_{1} & -s_{2}^{*} & \frac{s_{3}^{*}}{\sqrt{2}} & \frac{s_{3}^{*}}{\sqrt{2}} \\
s_{2} & s_{1}^{*} & \frac{s_{3}^{*}}{\sqrt{2}} & -\frac{s_{3}^{*}}{\sqrt{2}} \\
\frac{s_{3}}{\sqrt{2}} & \frac{s_{3}}{\sqrt{2}} & \frac{-s_{1}-s_{1}^{*}+s_{2}-s_{2}^{*}}{2} & \frac{s_{1}-s_{1}^{*}+s_{2}+s_{2}^{*}}{2} \\
\frac{s_{3}}{\sqrt{2}} & -\frac{s_{3}}{\sqrt{2}} & \frac{s_{1}-s_{1}^{*}-s_{2}-s_{2}^{*}}{2} & \frac{-s_{1}-s_{1}^{*}-s_{2}+s_{2}^{*}}{2}
\end{array}\right)
\end{aligned}
$$

The required orthogonality associated with adequate recombination makes it possible to obtain signals $\tilde{s}_{i}$ which only depend on the symbol $s_{i}$. This result can be seen, like the Alamouti code, as a diagonalization of the channel. In addition, the coefficient $\sqrt{\frac{P_{0}}{n_{T}}}$ makes it possible to control the power emitted. System using OSTBC is equivalent to $N_{s}$ independent SISO transmissions represented by Figure 2.

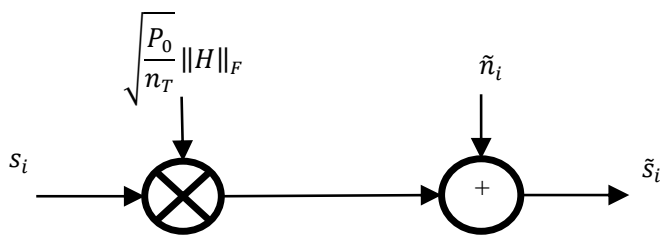

Figure 2. Equivalent SISO diagram of a system using OSTBC with the same gain for each channel

The strength of the code is to obtain a constant gain proportional to $\|H\|_{F}$ without a priori knowledge and to ensure a maximum order of diversity $n_{T} \times n_{R}$. The price to pay is a drop in flow with a yield $\mathcal{R} \leq 1$ corresponding to a spectral efficiency of $\mathcal{R} \log _{2} M$.

\subsection{Linear Precoding}

This family groups together the linear precoders whose difference with the codes presented until now is the need to know the channel on transmission. On transmission, a linear precoder in the form of a matrix makes it possible to combine the symbols to be transmitted and to distribute the power on the antennas according to a very precise strategy. On reception, a linear decoder processes the samples received. In general, the precoder-decoder couple optimizes a relevant criterion such as maximizing the capacity, maximizing the post-processing SNR or maximizing the minimum Euclidean distance. Each optimization gives its own result, but all these techniques diagonalize the channel.Tthe matrix equation of the model:

$$
Y=G H F S+G n
$$

With $s[b \times 1]$ the vector of symbols to be sent, $F\left[n_{T} \times b\right]$ the precoding matrix, and $G\left[b \times n_{R}\right]$ the decoding matrix. The first step of the reasoning consists in decomposing the precoding and decoding matrices in the form:

$$
F=F_{v} F_{d} \quad \text { and } \quad G=G_{d} G_{v}
$$

The matrices $F$ and $G$ are the solutions of optimization according to a criterion. The problem is now split into two: the couple $\left(F_{v}, G_{v}\right)$ makes it possible to obtain a virtual diagonal channel $H_{v}$ while the couple $\left(F_{d}, G_{d}\right)$ optimizes the desired criterion but by considering a diagonal channel of controlled dimension corresponding to $b$ channels with AWGN noise. We will see in the following, that the optimization is then simplified. This approach is illustrated by the block diagram in Figure 3.

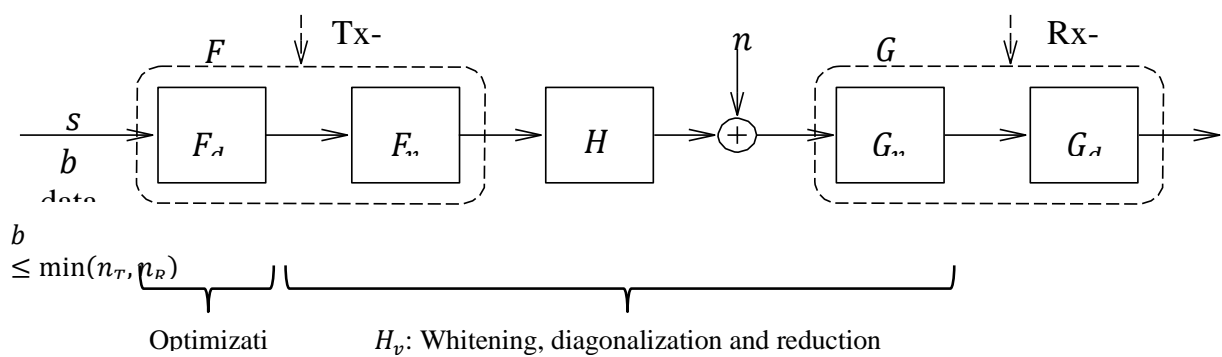

Figure 3. Block diagram of linear precoder in the virtual channel 


\subsection{Genetic Algorithm}

This evolutionary approach is based on the theory of evolution which explains the origins of biodiversity; species evolve over time to adapt to their environment, the most able individuals being more likely to survive and to transmit their characteristics. Over the generations, the accumulated changes give birth to new and increasingly specialized forms of life.

Genetic algorithms manipulate a population of individuals made up of solutions to a given problem. They evolve this population over several generations until they converge on an acceptable solution. The operations carried out during this evolution are directly modeled on biological terminology [15]:

\subsubsection{Selection}

This operation makes it possible to choose the individuals who will serve as the basis for building a new generation, the idea being to favor those who are doing the best. Deterministic selection, selection by tournaments, or even proportional selection are just a few variations of this operation.

- Deterministic selection: consists of choosing the $N$ best individuals.

- Selection by tournament: consists of randomly drawing $k$ individuals and choosing the best of them.

- Proportional selection: the number of times an individual is chosen is proportional to their fitness.

\subsubsection{Reproduction}

This operator makes it possible to directly replicate the $k$ best individuals of the current population to the next population. It allows on the one hand to maintain the presence of the best individuals throughout the iterations, and on the other hand, it allows to center the research on them.

\subsubsection{Crossover}

This operator makes it possible to combine and mix the genetic material present in the population. It can be seen as a local search (intensification) since it does not create new information. Formally, this consists of randomly selecting different parts of $p$ individuals and combining them to create one or more new individuals.

\subsubsection{Mutation}

This operation takes as an individual parameter, randomly selects a part, and makes a random modification. It is a disruptive element which removes the risk of premature convergence and extends the ability of the algorithm to explore the search space.

\subsubsection{Fitness}

The fitness of an individual is equivalent to his ability to solve a given problem. Generally, it is a function defined in $R^{+}$; the higher it is, the more the individual is able. The fitness function is the most important part of a genetic algorithm; it conditions both the nature of the solutions produced and the convergence time. One of the most powerful optimization approaches is genetic algorithms. They lend themselves well to parallelization and are used as a black box which adapts easily to most problems. It should however be noted that a bad configuration can lead to premature convergence, just as a bad conception of the fitness function can induce a significant complexity.

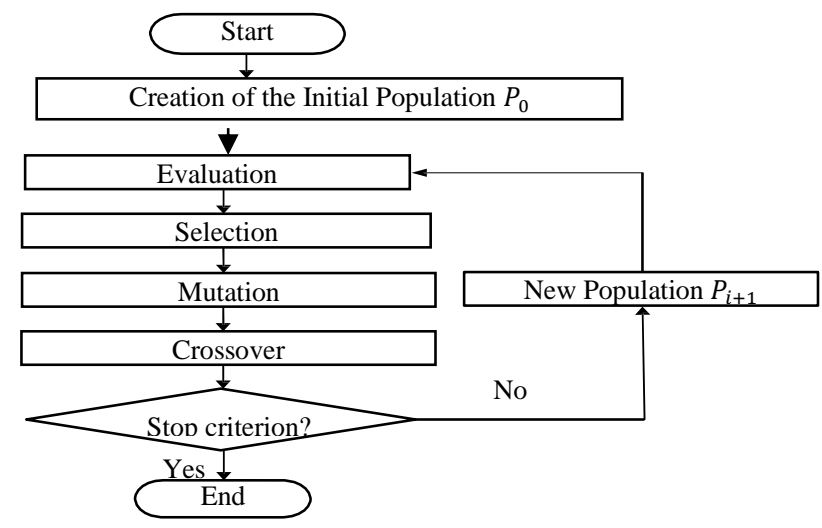

Figure 4. Flow diagram of a genetic algorithm

\section{RESULTS AND DISCUSSION}

The simulation was carried out in MATLAB environment and results are exhibited in this section. Figure 5 shows the performance comparison of Alamouti, Precoded Alamouti and the proposed Genetic Algorithm Optimized Precoded Alamouti schemes using ZF equalizer. The X axis indicates the SNR(dB) and the $\mathrm{Y}$ axis is the logarithmic representation of BER. It can be clearly interpreted from the plots that the GA 
optimization Precoded Alamouti outperforms the basic Alamouti coding scheme and the precoded Alamouti coding scheme mentioned in XingwangLi et al.[11]

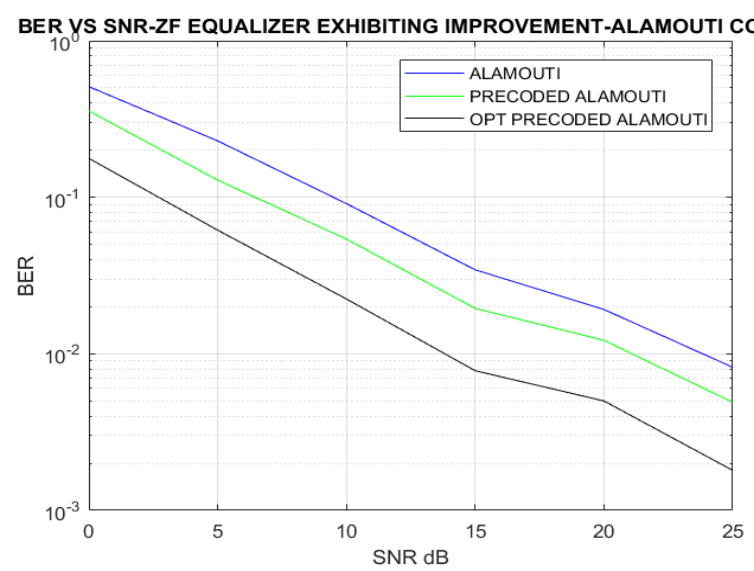

Figure 5 . Performance comparison of Alamouti, Precoded Alamouti [11] and proposed GA-optimized Precoded Alamouti schemes using ZF equalizer.

It is evident from figure 5 and its respective Table 1, that an the proposed Genetic Algorithm Optimization of the precoded Alamouti codes using ZF equalizer results in a considerable decrease in the BER as the SNR increases. It is also found that the BER achieved is decreased approximately by $1 / 3$ of the value of BER achieved using Alamouti Codes.

Table 1. Comparison of SNR vs BER using ZF Equalizer between the proposed algorithm and previous schemes

\begin{tabular}{llll}
\hline $\begin{array}{c}\text { SNR } \\
(\mathrm{dB})\end{array}$ & \multicolumn{3}{c}{ BER Achieved Using ZF Equalizer } \\
\cline { 2 - 4 } & Alamouti code & Precoded Alamouti code [11] & $\begin{array}{l}\text { Proposed } \\
\text { Precoded Alamouti Code }\end{array}$ \\
\hline 0 & $4.9 \times 10^{-1}$ & $3.6 \times 10^{-1}$ & $1.8 \times 10^{-1}$ \\
5 & $2.2 \times 10^{-1}$ & $1.3 \times 10^{-1}$ & $5.4 \times 10^{-2}$ \\
10 & $9.2 \times 10^{-2}$ & $5.1 \times 10^{-2}$ & $2.1 \times 10^{-2}$ \\
15 & $3.9 \times 10^{-2}$ & $1.9 \times 10^{-2}$ & $8.6 \times 10^{-3}$ \\
20 & $1.7 \times 10^{-2}$ & $9.4 \times 10^{-3}$ & $3.3 \times 10^{-3}$ \\
25 & $9.1 \times 10^{-3}$ & $4.7 \times 10^{-3}$ & $2.3 \times 10^{-3}$ \\
\hline
\end{tabular}

Figure 6 shows the performance comparison of Alamouti, Precoded Alamouti and proposed Genetic Algorithm Optimized Precoded Alamouti schemes using MMSE equalizer. The X axis indicates the SNR(dB) and the $\mathrm{Y}$ axis is the logarithmic representation of BER. It can be clearly interpreted from the plots that the GA optimization of Precoded Alamouti outperforms the Alamouti coding scheme and precoded Alamouti coding scheme mentioned in Jiang et al.[12].

The suggested Genetic Algorithm Optimization of the precoded Alamouti codes utilising MMSE equalisation leads in a significant drop in the BER as the SNR improves, as shown in figure 6 and its related tabulated results in Table 2 .

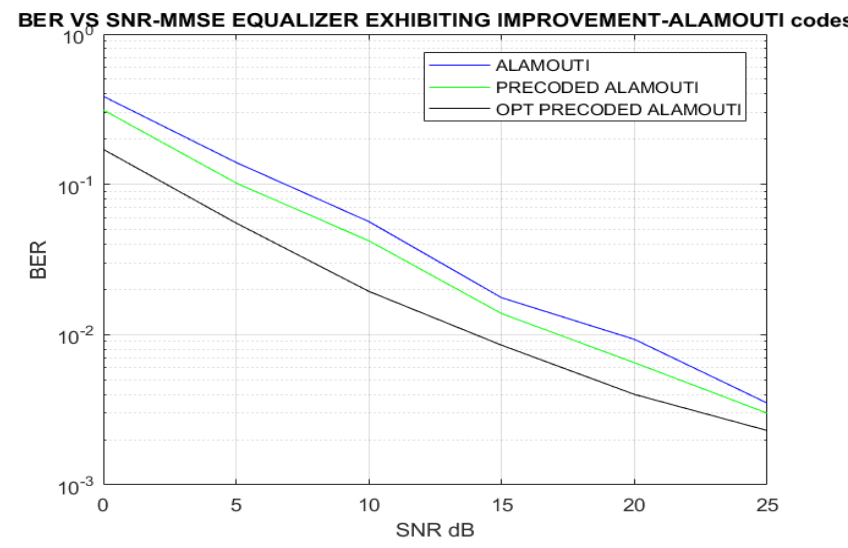

Figure 6. Performance comparison of Alamouti, Precoded Alamouti [12] and proposed GA-optimized

Precoded Alamouti schemes using MMSE equalizer. 
Table 2. Comparison of SNR vs BER using MMSE Equalizer between the proposed algorithm and previous schemes

\begin{tabular}{llll}
\hline $\begin{array}{l}\text { SNR } \\
(\mathrm{dB})\end{array}$ & \multicolumn{3}{c}{ BER Achieved Using MMSE Equalizer } \\
\cline { 2 - 4 } & Alamouti code & Precoded Alamouti code [12] & $\begin{array}{l}\text { Proposed } \\
\text { Precoded Alamouti Code }\end{array}$ \\
\hline 0 & $3.8 \times 10^{-1}$ & $3.1 \times 10^{-1}$ & $\begin{array}{c}\text { Optimized } \\
5\end{array}$ \\
10 & $1.4 \times 10^{-1}$ & $1.0 \times 10^{-1}$ & $5.4 \times 10^{-1}$ \\
15 & $5.3 \times 10^{-2}$ & $4.3 \times 10^{-2}$ & $1.7 \times 10^{-2}$ \\
20 & $2.5 \times 10^{-2}$ & $1.4 \times 10^{-2}$ & $7.1 \times 10^{-3}$ \\
25 & $9.6 \times 10^{-2}$ & $7.7 \times 10^{-3}$ & $2.8 \times 10^{-3}$ \\
\hline
\end{tabular}

Using the ZF equaliser, Figure 7 compares the performance of OSTBC, Precoded OSTBC, and proposed Genetic Algorithm Optimized Precoded OSTBC schemes. The graphs clearly show that the GA optimization of Precoded OSTBC outperforms the OSTBC coding scheme and the precoded OSTBC coding scheme described by XingwangLi et al.[11]

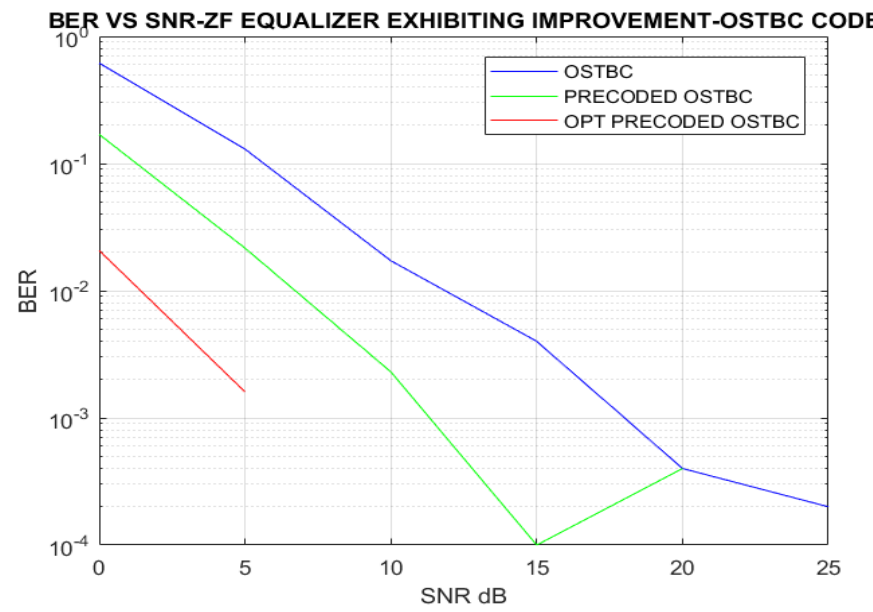

Figure 7. Performance comparison of OSTBC, Precoded OSTBC [11] and proposed GA-optimized Precoded OSTBC schemes using ZF equalizer.

Figure 7 and its corresponding tabulated findings in Table 3 indicate that optimising the precoded OSTBC codes using ZF equalisation results in a significant drop in the BER as the SNR improves. It is also evident from the results that OSTBC codes yield better results than Alamouti codes.

Table 3. Comparison of SNR vs BER using ZF Equalizer between the proposed algorithm and previous schemes

\begin{tabular}{llll}
\hline \multirow{2}{*}{$\begin{array}{l}\text { SNR } \\
(\mathrm{dB})\end{array}$} & \multicolumn{3}{c}{ BER Achieved Using ZF Equalizer } \\
\cline { 2 - 4 }
\end{tabular}

The simulation results in Figure 8 show the performance comparison of OSTBC, Precoded OSTBC and proposed GA-optimized Precoded OSTBC schemes using MMSE equalizer. It can be clearly interpreted from the plots that the GA optimization of Precoded OSTBC outperforms the precoded OSTBC scheme mentioned in Jiang et al.[12] and the results are listed in Table 4. 


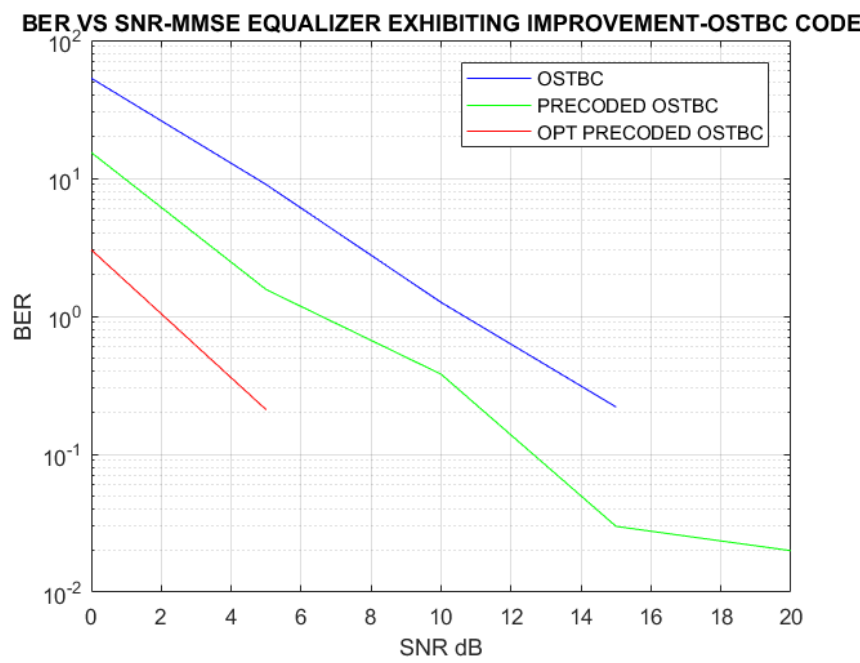

Figure 8. Performance comparison of OSTBC, Precoded OSTBC [12] and proposed GA-optimized Precoded OSTBC schemes using MMSE equalizer.

Table 4. Comparison of SNR vs BER using MMSE Equalizer between the proposed algorithm and previous schemes

\begin{tabular}{llll}
\hline \multirow{2}{*}{$\begin{array}{l}\text { SNR } \\
(\mathrm{dB})\end{array}$} & \multicolumn{2}{c}{ BER Achieved Using MMSE Equalizer } \\
\cline { 2 - 4 } & OSTBC code & Precoded OSTBC Code[12] & $\begin{array}{l}\text { Proposed } \\
\text { Precoded OSTBC Code }\end{array}$ \\
\hline 0 & $5.3 \times 10^{-1}$ & $1.5 \times 10^{-1}$ & $3.0 \times 10^{-2}$ \\
5 & $9.0 \times 10^{-2}$ & $1.6 \times 10^{-2}$ & $2.1 \times 10^{-3}$ \\
10 & $1.3 \times 10^{-2}$ & $3.8 \times 10^{-3}$ & 0 \\
15 & $2.2 \times 10^{-3}$ & $3.0 \times 10^{-4}$ & $3.0 \times 10^{-4}$ \\
20 & 0 & $2.0 \times 10^{-4}$ & 0 \\
25 & 0 & 0 & 0 \\
\hline
\end{tabular}

\section{CONCLUSIONS}

From the above obtained results, the following points can be concluded:

(i) The proposed Genetic Algorithm Optimization of the precoders using Alamouti/ OSTBC codes result in an overall decrease of Bit Error Rate with increase in SNR thereby increasing the efficiency of the Single User MIMO system.

(ii) Results of MMSE equalizer are better than $\mathrm{ZF}$ equalizer results.

(iii) The OSTBC codes give better results than the Alamouti codes.

\section{REFERENCES}

[1] Deepak H. A., T. Vijayakumar, Optimal Threshold Estimation using Grey Wolf Optimization for EMD-DTCWT Based ECG Denoising" , International Journal of Recent Technology and Engineering, 2020

[2] Sindhwani, N., Bhamrah, M.S., Garg, A. and Kumar, D., 2017, July. Performance analysis of particle swarm optimization and genetic algorithm in MIMO systems. In $20178^{\text {th }}$ International Conference on Computing, Communication and Networking Technologies (ICCCNT) (pp. 1-6). IEEE.

[3] Mitchell, B., 2015. Wireless Standards 802.11 a, 802.11 b/g/n, and 802.11 ac. Verkkojulkaisu. Saatavissa: http://compnetworking. about. com/cs/wireless80211/a/aa80211standard. htm [viitattu 8.4. 2015].

[4] Lagrange, X., 2014, April. Very tight coupling between LTE and Wi-Fi for advanced offloading procedures. In 2014 IEEE Wireless Communications and Networking Conference Workshops (WCNCW) (pp. 82-86). IEEE.

[5] Wannipa, Y., 2008. A Study on the Performance of Compact MIMO Antenna for Portable Terminals.

[6] Grover, A. and Grover, N., 2014. On limits of wireless communications in a fading environment: a general parameterization quantifying performance in fading channel. Indonesian Journal of Electrical Engineering and Informatics (IJEEI), 2(3), pp.125-131.

[7] Larsson, E.G. and Stoica, P., 2008. Space-time block coding for wireless communications. Cambridge university press.

[8] Alamouti, S., 1998. A simple diversity technique for wireless communications. IEEE JSAC, 16, pp.1451-1458.

[9] Tarokh, V., Seshadri, N. and Calderbank, A.R., 1998. Space-time codes for high data rate wireless communication: Performance criterion and code construction. IEEE transactions on information theory, 44(2), pp.744-765.

[10] Tarokh, V., Jafarkhani, H. and Calderbank, A.R., 1999. Space-time block codes from orthogonal designs. IEEE Transactions on Information theory, 45(5), pp.1456-1467. 
[11] Xingwang Li, Xueqing Yang, Lihua Li, Jin Jin, Ning Zhao, Changsen Zhang, "Performance Analysis of Distributed MIMO with ZF Receivers over Semi-Correlated K Fading Channels", IEEE. Translations, IEEE Access ,DOI 10.1109/ACCESS.2017.2705146, 2016.

[12] Yi Jiang, Mahesh K. Varanasi, Jian Li, "Performance Analysis of ZF and MMSE Equalizers for MIMO Systems: An In-Depth Study of the High SNR Regime", IEEE Transactions on Information Theory, Vol. 57, No. 4, April 2011

[13] Bibhuti Bhusan Pradhan, Lakshi Prosad Roy, "Ergodic capacity and symbol error rate of distributed massive MIMO systems over Rayleigh-inverse Gaussian fading channels using ZF detectors", Physical Communication 38, Elsevier B V, , https://doi.org/10.1016/j.phycom.2019.100906, 2020.

\section{BIOGRAPHY OF AUTHORS}

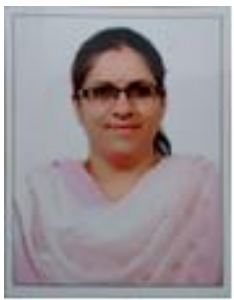

Ms Harsha Gurdasani is a Research Scholar in Department of Electronics and Communication Engineering, JAIN(Deemed -to-be University), Bangalore., INDIA. She is purusing her doctoral degree in the field of Wireless Communication. Having $13+$ years of teaching experience in Electronics \& Communication Engineering, The author has delivered 15 papers at national and international conferences and has published seven papers in foreign publications.

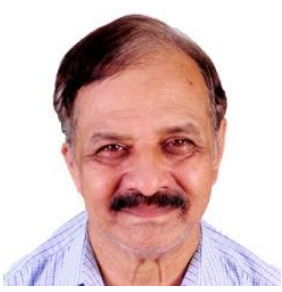

Dr. A G Ananth, presently is a Professor in the Department of Electronics \& Communication Engineering, NMAMIT, Nitte, Mangalore, INDIA. Former ISRO Dy. Director, ISRO RESPOND programme having 10 years of research experience in Space Physics, 25 Years of Industry R\&D experience at ISRO Bangalore and 10 Years of Teaching expeience in Electronics and communication engineering. He has published 15 Research papers in Space Physics and 35 Reseach papers in communication and related areas of engineering in International Journals. He has Completed 500 R\&D projects relating to space technology and applications in ISRO, Bangalore.

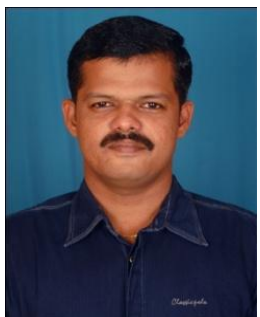

Dr. Thangadurai. $\mathrm{N}$ is working as Professor and Head of the Department of Electronics and Communication Engineering, JAIN (Deemed-to-be University) Bangalore. He has obtained his Ph.D Degree in Wireless Sensor Networks from Bharathiar University, Coimbatore. He has obtained his Bachelor's Degree in Electronics and Communication Engineering from Coimbatore Institute of Technology and Master's Degree in Applied Electronics from Mohamed Sathak Engineering College under Anna University. He has published 119 research papers in both International and National Journals and Conferences. He has supervised 64 numbers of undergraduate and postgraduate students for their project completion and guiding $8 \mathrm{Ph} . \mathrm{D}$., scholars now in which one awarded in 2019. He is currently working with sponsored research project grant received from ISRO. He has also filed 23 Indian patents into his credit and 18 published. He also published 5 books into his credit. His research interests are Communication, Networking, Wireless Communication, Satellite Communications, Mobile Adhoc and Wireless Sensor Networks, VANET, Embedded Systems, Optical Communication, and Navigation Systems. He is also a Life member of following professional bodies like ISCA, ISTE, IAENG, and IACSIT. Also he is a fellow of IETE and OSI. He has received many awards like Best Young Scientist Award-2018, Award for Research Publications from VGST 2016-17, Bharat Excellence Award -2017, Outstanding Researcher Award -2016 . 